\title{
A Unique, Culture-Aware, Personalized Learning Environment
}

\author{
http://dx.doi.org/10.3991/ijet.v7iS2.2323 \\ T. Swinke \\ University of Applied Sciences Worms, Worms, Germany
}

\begin{abstract}
This paper examines what current learning systems offer towards the idea of a multi-dimensional learning system. It will show the requirements for a multidimensional learning system and that no current system is able to meet them. Therefore a new model is proposed that is not only capable of fulfilling the requirements for cultural diversity but also of satisfying the rising demand for personalization that has been rising in the course of the last twenty years. This new model will enable systems, which bring the personalization of e-learning to the next level.
\end{abstract}

Index Terms-adaptive, cultural ,e-learning, multidimensional, personalization, user profile

\section{INTRODUCTION}

The demand for life-long learning is increasing in importance in this fast paced world [35]. Learners have to gain more knowledge in less time. Employees are being required to continue learning as part of their everyday work life in order to stay with the fast changing business environment.

Due to the phenomenon of "academic inflation" as called by Sir Ken Robinson the importance of academic degrees is rising, because "you need an MA where the previous job required a $\mathrm{BA}$, and now you need a $\mathrm{PhD}$ for the other" [36], so there is a higher demand for academic degrees.

These conditions, together with factors such as spatial distance between learner and provider and need for asynchronous education have lead to the position that elearning has reached today. E-learning has also become quite ubiquitous throughout the learning environment, starting at elementary through high school up to higher education. Even international enterprises rely on interactive material, online classes and video tutorials.

Besides the higher need for e-learning, the beneficiaries of e-learning have changed [35]. In the past the typical learners that used the first forms of e-learning, e.g. Computer-Based-Training or Web-Based-Training, were homogeneous groups: A group of employees of the same company or the same industry/ a group of students within the same class or school, but this fact has changed.

Everybody wants to enjoy liberties and evolve on their own terms. Traditional e-learning was not targeted for those developments and is not able to cater to an individual. Beneficiaries of e-learning have become much more individual and diverse. Individuality has become a great factor in society and the demand for it has been rising during the last twenty years [27]. Most learning systems are targeted towards either a single anonymous learner or a clustered group of learners (one-size-fits-all approach).
In addition, diversity has changed over the last years. Most corporations have not only sites in different countries, but also diversification in almost every cultural, national and religious background [35].

Universities have more international students then ever; up to $74 \%{ }^{1}$. In the United States the increase of international students in general went up 5\% in 2010/20112. These numbers are only representing active students on campus.

For example the number of international students only from China went up 28\% in 2011 [6]. Enrollment in elearning is creating a new impact on diversity within higher education.

In general e-learning beneficiaries are become more individual, more multi-cultural, and more diverse. So it becomes more clear that the traditional one-size-fits-all method of the traditional e-learning is not a suitable approach.

\section{PROBLEM}

The strongest influences on the learners' success are his learner preferences, interests and emotions, in other words his personal characteristics towards learning.

A lecturer can easily spot the mental state of his students empathically and react on it by explaining terms specifically for some students or choosing a different example, or even just repeat some content. But it is impossible for the lecturer to target each and every student individually, because he simply does not have the time or the resources.

Digital material has been used to support the learning process for quite some time now [7]. However, the existing systems are mostly using low interaction with the learner. Newly developed features and technologies available today are rarely used to max out the potential of these new technologies.

Most popular Learning Management Systems (LMS), like Moodle ${ }^{3}$ and OLAT $^{4}$ have been developed for homogenous target groups with unattributed learners. They pose as a combination of web pages and document management systems but completely lack the possibility to cater to any special need a learner might have [21]. Every single learner in the same class is served the same content

\footnotetext{
${ }^{1}$ http://www.thecompleteuniversityguide.co.uk/ international/international-students-the-facts/by-university

${ }^{2}$ http://www.iie.org/en/Who-We-Are/News-and-Events/PressCenter/Press-Releases/2011/2011-11-14-Open-Doors-International$\underline{\text { Students }}$

${ }^{3}$ http:/www.moodle.org/

${ }^{4}$ http://www.olat.org/
} 
despite their personality, their preexisting knowledge, or their cultural background: the classic "one size fits all" approach.

Bloom [4] showed that a one-on-one tutoring is the most effective way to teach students. Since the demand for academic degrees have increased dramatically, it is not feasible to get a tutor for every student. A computer could never replace a human tutor, but a personalized system could still lead to better learning results than a traditional one.

The traditional approach of only providing learning material, lacks of the most important advantage any computer-based system brings: computing power. The one advantage computers have is their ability to compute much faster than humans. So adapting learning content automatically to the learners needs would be an easy task, if there were a model that would provide rules how to adept what content to what learner.

\section{Present ApProaches}

In this section some of the approaches that target to create more meaningful learner profiles and better adapting systems are examined. Most of these systems only embrace one or two factors or they are not using the factors in a deep enough manner. The examined systems are grouped by their activity in the field of adaptivity, personalization, and cultural awareness.

\section{A. Adaptivity}

The term "adaptivity" has a wide range of meanings. In the context of this paper adaptivity is understood as the alignment of learner objects to the learners mental state as well as his actions. The result of the adaptation process will be seen as personalization.

A first level of adaptation can be reached by using a recommender system [38] to select valid learning items for the learner. Recommender systems can be used in three different approaches. The collaborative approach [38] compares the behavior of similar learners and which items the use, the content-based [38] approach examines the characteristics of the items a learner used in the past. The third approach is the hybrid approach [25], which is a combination of the aforementioned.

A problem that has to be faced is that all recommender systems suffer from the "cold start problem" which means that the system needs a certain amount of information about the learner's and items in order to work properly.

A second way of adaptation is changing of navigation according to the learner's actions like ELM-ART [46] or InterBook [2]. The constraint here besides the "cold start problem" is the limited knowledge about the learner. The system can react on the learner's decisions, but the learner has to fail in order for the system to adept.

Another way of adaption is the actual manipulation of content in any form. The system [5] is suggesting actually replaces pictures within the content accordingly to the learners cultural background.

\section{B. Personalization}

First tests are created in order to determine a general group or cluster a learner fits into, in order to cater to the needs of his group. The learner type test by [37] e.g. measures the learners stamping of eight categories and tries to decide which instructional model fits best.

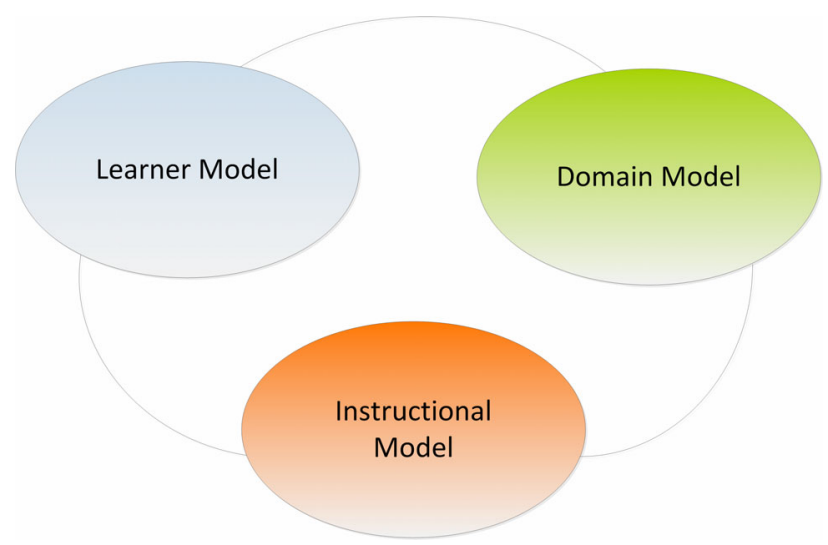

Figure 1. The three parts of a model

The downside of this proceeding is that learners can have the same values in all six categories but be completely different in other characteristics that are not tested.

\section{Cultural Awareness}

The cultural background of a student is mostly underestimated although it is marked as very important for the learning process [39]. Very few LMS embrace the cultural background of learners. Blanchard et al. [5] are using the cultural background of learners within their system, but only define the cultural background by the learners' nationality. Simply the nationality of the learner is not sufficient any more. People have more intercultural influences, travel more and move across the entire world, so there has to be a new measurement.

\section{REQUIREMENTS FOR A COMBINED MODEL}

As the previous chapter shows there are already a lot of approaches for adaptive systems. This chapter will show requirements for a merged system thereby examining if the already existing approaches can be combined.

In order to build an efficient adaptive multi-dimensional model, there are three basic questions that need to be answered [44]:

1. What will be adapted?

2. To who will be adapted?

3. How will be adapted?

Therefore [44] describes a model as a combination of three parts that can be seen in figure 1: The domain model (what), the learner model (who), and the instructional model (how). The requirements of these three parts are described in more detail within this section.

A fourth part would be the presentation part, which answers the question how the results will be displayed. The presentation part will not be part of this examination.

\section{A. Requirements for a learner model}

In order to provide the optimal basis for adaptation, the learner model has to have a learner profile as accurate as possible. Thus besides the history of the learners' actions, the model needs to record a set of personality traits.

Since it is very important for learners to interact with other learners while learning [45], [9], the system has to perceive the learner as a social being and thus be able to discover the learners' social interactions. For a cultural aware adaptation the learner model needs to assess the cultural background instead of the nationality of the learner. In addition the learner model has to be able to 
detect long-term and short-term changes within the learners characteristics.

\section{B. Requirements for a domain model}

For the instructional model, which is described in the next section, to work properly, the domain model should hold the learning objects (content) in a structured manner. Besides the learning objects that have been created by lecturers it has to contain learning objects created or imported by learners. The importing process should not be limited to certain file types but should be as ubiquitous as possible. In order to have a rich domain model it has to be easy for the learner to import and enrich the content. If a learner adds content, the created learning object has to be tagged with the learners' profile.

The learning objects have to be as fragmental as possible. The smaller the learning objects are defined the more fine grained the content can be adapted to the learners needs.

As a very important point the relationships between the learning objects have to be stored in a structured manner as well. The correlations like exclusive, inclusive, or parallel have to be clearly defined.

\section{Requirements for a instructional model}

The target of the instructional model is not to accomplish the most adaptation, it is more important to accomplish meaningful adaptation.

In order to reach an optimal adaptation the following adaptation concepts should be supported:

Adaptive Course Delivery enables the model to suggest or select distinctive learning objects or series of learning objects according to the learners' profile. This helps the learner to learn especially towards a previous defined learning target.

Content Discovery and Assembly facilitates the model to connect to various sources of learning content and discover available learning objects and thus assemble learning objects tailored to the learners needs.

Adaptive Collaboration Support empowers the model to see the learner not as a single person but as a part of a virtual learning community. It can suggest learning group formations, tutoring relationships between learners or act as an agent to contact a lecturer. It can even act as a rapid alert system for learners that are about to give up.

The main task of the instructional model is twofold: The adaptation logic part analysis if the learner needs adaptation at a certain point, which is done by a matching of all the learners characteristics, his performance and the possible learner objects available.

The adaptation actions define which learning objects are available or composable and adapt them to the learners' profile. They also provide collaboration options or instructional support.

Another requirement for the instructional model is that the model has to be able to store rules and guidelines for pedagogical instructional models, which then can be predefined by an instructor or the learning object.

\section{Proposal For A Multi-Dimensional Model}

As one can reason by the means of the previous requirements the existing approaches cannot meet all of them.

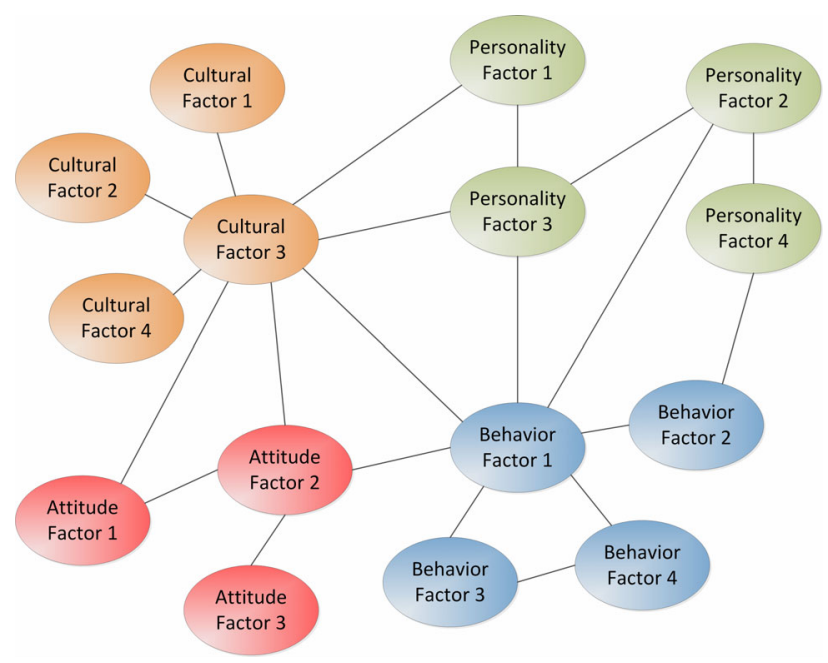

Figure 2. Example of mutual impact of factors of the learner model

Various functions can already be delivered by existing approaches as mentioned above; others could be improved or added. The measurement of the cultural background for example is not widely spread and could be an immense improvement for adaptive systems. The assessment of more detailed personality characteristics could lead to a better-personalized content.

Other functionalities are not considered yet at all within the examined systems. The possibility for learners to import not just content from other learning systems in a standard format, but import every content from tools they use every day anyway like YouTube ${ }^{5}$ or Twitter ${ }^{6}$ are not given yet. Also tagging the imported content anonymously, using the learner's characteristics, could improve the content eminently. This tagging could by used by the instructional model to find learning objects for a learner that has similar characteristics than the learner that added the content.

In addition the social behavior within a learning system should be picked up for offering the learner contacts to other learners, forming learning groups or offering tutoring help from other learners or lecturers.

A pooling of the examined approaches cannot achieve the desired outcome, caused by the lack of interfaces to the internal factors. All adaptive systems work with internal factors that influence each other; a pooled system would lack the possibility of one factor of one system influencing a factor from another system directly as depicted in figure 2. So for example a correlation between a factor within the cultural background of the learner and a factor within learners' behavior could not be detected since there is no direct connection.

In order to provide this ability of mutual interaction and the chance to evaluate correlations between factors, an integrated system based on a multi-dimensional model is needed. The different dimensions are described in more detail in the following sections.

\section{A. Learner model}

The learner model has to contain mechanisms for understanding what the student does, how he thinks and what he feels. This can be described by learner character-

\footnotetext{
${ }^{5}$ http://www.youtube.com

${ }^{6}$ http:/www.twitter.com
} 
istics that were gathered from previous held assessments and from logging and tracking the learner within the system. Shute and Towle [42] noted that the validity and reliability of the assessment are crucial for a sound learner model.

The more detailed and more tailored to the needs the learner profile, the more accurate the content can be adapted to the learners needs [32].

In order to get the most detailed learner profile possible, the assessed characteristics are grouped into four different dimensions, as illustrated in figure 3 , that cater to different parts of the learner model:

\section{1) Cultural dimension}

There are numerous indications that the cultural background of a learner is massively influencing his learning attitude and his previous knowledge [39]. His learning success, preferences, interests and emotions are also being affected. Vygotski [45] even said that it is "a part of each person's nature".

By including this cultural background, the right examples and analogies can be selected to illustrate circumstances within the content. It can also help to create a better matching curriculum according to his needs. Also for the system to under stand the learners social interaction, the assessment of the cultural background is crucial.

There are several approaches available to measure cultural influences: [41], [29], [19], [8] with Hofstedes model [23] being the most popular.

Factors to describe the cultural dimension could include:

- Power Distance Indicator (PDI) [23]

- Individualism (IDV) [23]

- Uncertainty avoidance Index (UAI) [23]

- Masculinity (MAS) [23]

- Long term orientation (LTO) [20]

- Indulgence [20]

\section{2) Personality dimension}

Cultural background is not as accurate an indicator of learner behavior as the learner's personality. Theoretically, the learner's personality could differ considerably from that of his cultural peers. The social behavior and interaction preferences are also something that is included within the learner's personality.

The learner's personal preferences, learning preferences and characteristics could be assessed up by a personality test for instance: Meyer-Briggs [34], DISG [31], Big Five [14], or "Multiple Intelligences" [11].

Additional some factors independent from the personality test can be assessed:

- Working memory capacity [13]

- Intelligence [26]

- Learning style [16]

- Learning goals or goal orientation [26]

\section{3) Attitude dimension}

Repeating learning content that the learner already knows, can demotivate and frustrate the learner [15], [10]. So there has to be a possibility to assess what content the learner already mastered and remove it from online classes.

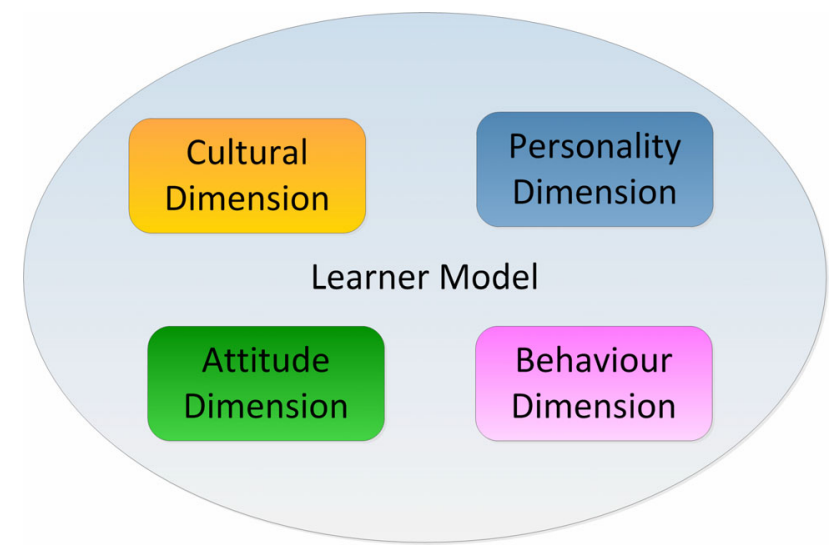

Figure 3. The four dimensions of the leaner model

- Self-efficiency [33]

- Prior knowledge [15]

- Mood [3]

- Frustration [15], [10]

- Delight [15]

- Confusion [15]

- Certainty [10]

\section{4) Behavior dimension}

In addition to the rules of the aforementioned adaptive e-learning systems, in order to adapt to the learner's performance, the system could pick up on general learner behavior - behavior like frequency of computer usage and session length.

The model does not only encapsulate general information about the learner, but can also be based on a detailed tracking and logging of the learner's behavior within the system. This tracking information can be used to detect changes within his behavior and inform a supervisor in certain cases.

- The degree of self-regulated learning [1]

- Reaction to Bonus / Badges

- Need for help or feedback [25]

- Number of Logins

- Need for Control [28], [43]

- Number of tries per task [22]

- Received grades [22]

- Exercises already made [22]

\section{B. Domain model}

The domain model contains the content or knowledge that is to be taught, as well as the relationships between the content elements. These elements could be created by a lecturer, a fellow learner or could even be imported from common data sources e.g. YouTube. Besides the relationships between the elements like hierarchies, some of the content can exist in pre-rendered variations in order to work faster with variations that are used often.

Elements should be stored in a well-established format (i.e. IMS in order to be able to import and export content.

The learning objects contain tags that can be set manually or can be automatically derived from the learner profiles in order to show which learners have been using what variation of learning objects. According to the learn- 
ers profile and the tags of the learning object, a version of the learning object is produced for the learner.

The elements can be used to select what instructional model would fit best to present it.

\section{Instructional model}

The instructional model contains the entire instructional strategy. Paramythis et al [30] also call this model the adaptation model that defines what can be adapted, and when and how it is to be adapted.

It contains all rules for adaptation, generating content variants and reacting on the learner behavior. It also contains all available educational methodology in order to adept content not only on means of the learner but also towards an educational strategy. Thus it is possible to determine what methodology the learner should follow.

The proposed instructional model will include several algorithms and models itself in order to deliver the best content variation.

One component will be a recommender system that consists of two parts:

The collaborative part can make use of extensive learner profiles to analyze similarities between learners and personalize learning content based on all learner model factors. The personalization can be effected right from the beginning, since a basic learner profile already exists. An additional rating system that allows the learner to rate content by his learning satisfaction or understanding with it, can also make use all four factors.

The content-based part of the system is able to obtain automatically a first rating of the person that included or created that content. The content procures the learners value from all four dimensions automatically. Over and above that, it is possible to have an automatic rating system that semantically rates the content by specified properties.

The main adapting engine could contain one form of artificial intelligence: Partial Least Squares (PLS), Neuronal Networks, Fuzzy Logic or Data Mining.

\section{CONCLUSION \& OUTLOOK}

The proposed model integrates very detailed information about the learner, his way of thinking and learning, his cultural background, his learning attitude and his behavior. It captures everything from a broad picture like the culture of a learner down to the narrow behavior he might show only temporary. It is offering a part to store learning content in different variations. Most importantly it provides a method to select, discover, compose and adapt the content respecting all factors that are assessed within the learners profile and the relationships between them.

The learner profile is highly complex and every factor is able to affect all other factors in order to achieve a most realistic representation of the learner. The learner is able to overrule the decisions of the model at all times and thus correct and refine the models decisions. These interventions cannot only be made by the learner himself, his tutor can also engage and change the course of the learner.

Since "the mechanism of individual developmental change is rooted in society and culture." [45] and following Siemens' Connectivism [40], there has to be a community notion within the model.
The next step within research will be to examine all dimensions in more detail and evaluate the model.

Of course, there are a large number of critical issues that need to be taken into account.

Cultural issues include a learner behaving in a manner, which is incompatible with his cultural background. Some content from cultures could be seen as offensive in other cultural backgrounds.

The model also depends on a certain performance. Due to the vast amount of influencing factors, the complexity of the model can increase dramatically. This complexity can render an implemented system impossible to work.

Social issues include the learner being excluded from learning groups due to his profile. E-bullying could be an issue the learner has to be protected from. Another issue is that the learner should not be feeling repressed by the system. Thus there always has to be a possibility to change the proposal of the system and choose a different content. For the community part of the system, it is important to limit the information within learner profiles.

In future research it is to examine what dependencies exactly occur with which learners in what cultural environments. It is estimated that the proposed multidimensional model will proof to enhance the support of learners considerable.

\section{REFERENCES}

[1] Azevedo, Roger: Using Hypermedia as a Metacognitive Tool for Enhancing Student Learning? The Role of Self-Regulated Learning. In: Educational Psychologist 40 (2005), Dezember, Nr.4, S. 199-209

[2] Brusilovsky, P. ; Eklund, J.: A study of user model based link annotation in educational hypermedia. In: Journal of Universal Computer Science 4 (1998), Nr. 4, S. 429-448

[3] Beal, C. ; Lee, H.: Creating a pedagogical model that uses student self reports of motivation and mood to adapt ITS instruction. In: Workshop on Motivation and Affect in Educational Software, in conjunction with the 12th International Conference on Artificial Intelligence in Education (2005)

[4] Bloom, B S.: The 2 sigma problem: The search for methods of group instruction as effective as one-to-one tutoring. In: Educational Researcher 13 (1984), Nr. 6, S. 4-16

[5] Blanchard, E ; Razaki, R: Cross-cultural adaptation of e-learning contents: A methodology. In: World Conference on E-Learning in (2005)

[6] Choudaha, Rahul ; Chang, Li: Trends in International Student Mobility / Research \& Advisory Services World Education Services. 2012. - Forschungsbericht

[7] Cross, Jay: An informal history of eLearning. In: On the Horizon 12 (2004), Nr. 3, S. 103-110

[8] DiStefano, Joseph J. ; Maznevski, Martha L. ; University of Western Ontario. Western Business School. Research and Publications: Measuring Culture in International Management : the Cultural Perspectives Questionnaire. 1995

[9] Freire, Paulo: Pedagogy of the oppressed. Continuum Intl Pub Group, 1970

[10] Forbes-Riley, K. ; Rotaru, M. ; Litman, D.J.: The relative impact of student affect on performance models in a spoken dialogue tutoring system. In: User modeling and user-adapted interaction 18 (2008), Nr. 1, S. 11-43

[11] Gardner, H: Multiple intelligences: New horizons. 2006

[12] Graesser, Arthur C. ; D’Mello, Sidney K. ; Craig, Scotty D. ; Witherspoon, Amy ; Sullins, Jeremiah ; McDaniel, Bethany ; Gholson, Barry: The Relationship between Affective States and Dialog Patterns during Interactions with AutoTutor. (2008), April

[13] Graf, S ; Liu, TC ; Chen, NS: Learning styles and cognitive traitsTheir relationship and its benefits in web-based educational systems. In: Computers in Human Behavior (2009) 
[14] Goldberg, LR: The structure of phenotypic personality traits. In: American psychologist (1993)

[15] Graesser, A C.: AutoTutor holds conversations with learners that are responsive to their cognitive and emotional states. In: EDUCATIONAL TECHNOLOGY-SADDLE BROOK THEN ENGLEWOOD CLIFFS NJ-47 (2007), Nr. 1, S. 19

[16] Germanakos, P. ; Tsianos, N. ; Lekkas, Z. ; Mourlas, C. ; Samaras, G.: Capturing essential intrinsic user behaviour values for the design of comprehensive web-based personalized environments. In: Computers in Human Behavior 24 (2008), Nr. 4, S. 1434-1451

[17] Hofstede, Geert ; Hofstede, Jan-Gert: Cultures and Organizations Software of the Mind. 2005 HK07] 47 (2007), Nr. 1, S. 19

[18] Germanakos, P. ; Tsianos, N. ; Lekkas, Z. ; Mourlas, C. ; Samaras, G.: Capturing essential intrinsic user behaviour values for the design of comprehensive web-based personalized environments. In: Computers in Human Behavior 24 (2008), Nr. 4, S. 1434-1451

[19] House, Robert J. ; Global Leadership and Organizational Behavior Effectiveness Research Program: Culture, Leadership, and Organizations. Sage Publications, Incorporated, 2004 (The Globe Study of 62 Societies)

[20] Hofstede, Geert ; Hofstede, Jan-Gert: Cultures and Organizations Software of the Mind. 2005

[21] Hauger, D ; Köck, M: State of the art of adaptivity in e-learning platforms. In: Workshop-Week: Lernen-Wissen-Adaption, Halle, Germany (2007)

[22] Hospers, M ; Kroezen, E ; Nijholt, A ; Akker, R op d. ; Heylen, D: An agent-based intelligent tutoring system for nurse education. Department of Computer Science University of Twente, Enschede, The Netherlands, 200

[23] Hofstede, Geert: Culture's Consequences: International Differences in Work Related Values. Newbury Park, 1980

[24] Jannach, D ; Zanker, M ; Felfernig, A ; Friedrich, G: Recommender Systems: An Introduction. Cambridge University Press, 2010

[25] Koutsojannis, C. ; Prentzas, J. ; Hatzilygeroudis, I.: A web-based intelligent tutoring system teaching nursing students fundamental aspects of biomedical technology. In: Engineering in Medicine and Biology Society, 2001. Proceedings of the 23rd Annual International Conference of the IEEE 4 (2001), S. 4024-4027 vol. 4

[26] Kelly, D. ; Tangney, B.: Incorporating learning characteristics into an intelligent tutor. In: Intelligent Tutoring Systems (2002), S. 729-738

[27] Lauder, Hugh: Education, globalization, and social change. Oxford University Press, USA, 2006

[28] Lawless, K.A. ; Brown, S.W.: Multimedia learning environments: Issues of learner control and navigation. In: Instructional Science 25 (1997), Nr. 2, S. 117-131

[29] Lee, K P.: Culture and its effects on human interaction with design. In: Unpublished doctoral thesis, University of Tsukuba (2001)

[30] Loidl-Reisinger, Alexandros P. ; Susanne: Adaptive learning environments and e-Learning standards. (2004), Februar, S. 1-14

[31] Marston, WM: Emotions of normal people. Psychology Press, $1928 \mathrm{http}: / / \mathrm{dx}$.doi.org/10.1037/13390-000
[32] Martins,AC.;Faria,L;Carvalho, C V. ; Carrapatoso, E: User modeling in adaptive hypermedia educational systems. In: Educational Technology \& Society 11 (2008), Nr. 1, S. 194-207

[33] Mcquiggan, S.W. ; Mott, B.W. ; Lester, J.C.: Modeling selfefficacy in intelligent tutoring systems: An inductive approach. In: User modeling and user-adapted interaction 18 (2008), Nr. 1, S. 81-123

[34] Myers, I B.: Introduction to Type. A Guide to Understanding Your Results on the Myers-Briggs Type Indicator. Palo Alto. California: Consulting Psychologists Press, 1998

[35] Nagy, Attila: E-Content. In: Bruck, Peter A. (Hrsg.) ; Karssen, Zeger (Hrsg.) ; Buchholz, Andrea (Hrsg.) ; Zerfass, Ansgar (Hrsg.): The Impact of E-Learning. Springer Berlin Heidelberg, 2005, S. 79-96

[36] Robinson, Ken: Ken Robinson says schools kill creativity. http://www.ted.com/talks/ken_robinson_says_schools_kill_creativ ity.html

[37] Röll, Franz-Josef: P ädagogik der Navigation: Selbstgesteuertes Lernen durch Neue Medien. Kopäd, 2003

[38] Ricci, F ; Rokach, L ; Shapira, B ; Kantor, P B.: Recommender Systems Handbook. Springer, 2010

[39] Shen, CY: The Impact of Cultural Factors in Distance Learning: A Reviewing Research. In: editlib.org

[40] Siemens, G: Connectivism: A Learning Theory for the Digital Age 2004. In: Accessible on the Internet: http://www.elearnspace.org/ Articles/connectivism.htm (access 2011-08-12) (2004)

[41] Schwartz, S H. ; Lehmann, A ; Roccas, S: Multimethod probes of basic human values. In: Adamopoulos, J (Hrsg.) ; Kashima, Y (Hrsg.): Psychology and Culture Context: Essays in Honor of Harry C. Triandis. SAGE Publications, 1999

[42] Shute, V. ; Towle, B.: Adaptive e-learning. In: Educational Psychologist 38 (2003), Nr. 2, S. 105-114

[43] Tennyson, Robert D.: Instructional control strategies and content structure as design variables in concept acquisition using computer-based instruction. In: Journal of Educational Psychology 72 (1980), Nr. 4, S. 525-532

[44] Vandewaetere, M ; Desmet, P: The contribution of learner characteristics in the development of computer-based adaptive learning environments. In: Computers in Human Behavior (2011)

[45] Vygotsky, L S.: Mind in society (M. Cole, V. John-Steiner, S. Scribner, \& E. Souberman, Eds.). (1978)

[46] Weber, G. ; Brusilovsky, P.: ELM-ART: An adaptive versatile system for Web-based instruction. In: International Journal of Artificial Intelligence in Education (IJAIED) 12 (2001), S. 351-384

[47] Young, M.:The Technical Writer's Handbook. Mill Valley, CA: University Science, 1989.

\section{AUTHOR}

T. Swinke is with the Department of Computer Sciences at the University of Applied Sciences in Worms, Germany and is researching social and cultural influences in e-learning. (E-mail: tillman.swinke@gmail.com).

This work was supported in part by the University of Applied Sciences Worms. Submitted, October, 15, 2012. Published as resubmitted by the author on November, 7, 2012. 